\title{
ANÁLISIS FILOSÓFICO-CONCEPTUAL DE LA FIGURA DEL NEOCONSTITUCIONALISMO COMO UNA CLASE DE IUSNATURALISMO
}

\author{
PHILOSOPHICAL-CONCEPTUAL ANALYSIS OF THE FIGURE \\ OF NEO-CONSTITUTIONALISM AS A KIND OF NATURAL LAW
}

Sebastián Chavarría Lara ${ }^{1}$

DOI: https://doi.org/10.5377//rd.v40i1.8908

\section{RESUMEN.}

Es un lugar común en las discusiones iusfilosóficas de nuestra época, afirmar que el neoconstitucionalismo se distingue de manera importante del tradicional derecho natural. El objetivo de esta investigación trabajo consiste en desvanecer las tesis que sustentan dicha creencia. Se sostiene, en cambio, que el neoconstitucionalismo puede ser entendido como una especie de la gran familia de iusnaturalismos que han aparecido a lo largo de la historia de la cultura jurídica occidental. Y, respecto del vetus iusnaturalismo, se demuestra que el neoconstitucionalismo se distingue únicamente por vía de las diferencias específicas dentro del genus iusnaturalismo.

PALABRAS CLAVE: iusnaturalismos, neoconstitucionalismo, neopositivismo jurídico, vetus iusnaturalismo.

\begin{abstract}
:
It is a common place in the philosophical discussions of our time, to affirm that neo-constitutionalism distinguishes itself in an important way from traditional natural law theory. The aim of this work is to vanish the theses that support this belief. It is argued, on the other hand, that neo-constitutionalism can be understood as a kind of the great family of natural law theories that have appeared throughout the history of Western legal culture. And, with respect to vetus iusnaturalism, it is shown that neo-constitutionalism is distinguished only by way of specific differences within the genus iusnaturalism.
\end{abstract}

KEY WORDS: iusnaturalisms, neo-constitutionalism, legal neopositivism, vetus iusnaturalism.

Fecha de recepción: 02 de agosto del 2019

Fecha de aprobación: 07 de octubre del 2019

\footnotetext{
${ }^{1}$ Abogado. Maestrando en Filosofía del Derecho por la Universidad de Buenos Aires. Docente del Departamento de Teoría e Historia de la Universidad Nacional Autónoma de Honduras. Docente en el Centro Universitario Tecnológico Correo: $\underline{\text { schavarria@unah.edu.hn }}$
} 


\section{INTRODUCCIÓN}

Es un lugar común en las discusiones sobre filosofía del derecho de nuestra época, afirmar que el neoconstitucionalismo se distingue de manera importante del tradicional derecho natural. El objetivo de este trabajo consiste en poner de manifiesto que esto es menos cierto de lo que parece. De forma tal que consideramos que esta discusión está inscrita contemporáneamente dentro de lo que se ha venido en denominar el iusmoralismo (García Amado, 2015) y el vetus iusnaturalismo.

Ha sido particularmente entre los neoconstitucionalistas (o, postpositivistas como prefiere Atienza (Atienza, 2016) ${ }^{2}$ distinguirse claramente del iusnaturalismo tradicional, o viejo iusnaturalismo. Incluso, un positivista como Juan Antonio García Amado (2015), parece validar esa distinción a quien basta leerle en los siguientes términos:

El conjunto de doctrinas o autores que, para abreviar, podemos llamar antipositivistas no deben ser catalogados como iusnaturalistas, al menos si nos atenemos a un concepto preciso e históricamente avalado de iusnaturalismo. Efectivamente, yo a ti [a Manuel Atienza] no te tildaría de iusnaturalista, como tampoco pondría ese calificativo a otros grandes teóricos no positivistas, como Dworkin o Alexy. Tal vez podemos acordar que iusnaturalistas son los que afirman que existe el Derecho Natural y forma parte esencial de cualquier sistema jurídico, esté o no reflejado en las normas positivas del sistema (p. 230). (Lo resaltado es añadido).

En ese mismo sentido, García Amado (2015) afirma su desconcierto con respecto a los contenidos actuales del que él denomina iusmoralismo y lo compara críticamente con los iusnaturalistas, afirmando que:

\footnotetext{
${ }^{2}$ En este sentido, Atienza afirma que es distinto su constitucionalismo postpositivista del neoconstitucionalismo en tanto que habría algunas diferencias entre ambas teorías que permitiría inscribir a distintos autores en cada una de ellas sin confundirlas. Nosotros partimos de una base distinta. Se sume la premisa que tendrá será vindicada en este trabajo: que el neoconstitucionalismo (o postpositivismo) es una especie de iusnaturalismo.
}

Yo, positivista en cuanto a la Teoría del Derecho, entiendo el viejo iusnaturalismo, aunque no lo comparto. El iusnaturalismo nos dice qué es y dónde está el derecho natural. A los actuales iusmoralistas os entiendo peor, ya que no consigo dar con vuestra explicación sobre dónde están y por qué son Derecho (sentado que, para vosotros, no son derecho natural) y cómo pueden conocerse en sus contenidos esos principios morales que a la vez son plenamente jurídicos, y que son tantos y tan claros como para que el Derecho no tenga lagunas y para que pueda suponerse para cada caso difícil una única solución correcta (p. 243).

En esa misma discusión, resulta interesante que sea el mismo Manuel Atienza (2015) quien, a nuestro parecer, nos da ya un indicio de la cercanía que tiene este neoconstitucionalismo con respecto al vetus iusnaturalismo, citando a Aristóteles como el primer postpositivista:

Aunque mi manera de ver las cosas -el Derechose acerca, sin embargo, bastante (digamos, en lo que a aproximación metodológica se refiere) a la de Aristóteles. En mi Curso de argumentación jurídica, y basándome en parte en trabajos de Jesús Vega, incluí, provocativamente, un texto que titulé así: «¿Fue Aristóteles el primer postpositivista?». En algún sentido, yo creo que sí (p. 244). (Lo resaltado es añadido).

Esta última expresión nos da un primer indicio de que nuestra tesis es verdadera, pues los vetus iusnaturalistas se basan primordialmente en Aristóteles: si Aristóteles es el protopostpositivista, entonces esto nos da pie para sospechar que entre el neoconstitucionalismo (en tanto postpositivismo) y el viejo iusnaturalismo hay más coincidencias que diferencias (lo contrario de lo que tratan de poner de manifiesto los neoconstitucionalistas, con énfasis de distinguirse de cualquier escuela aristotélica tomista). Y eso es lo que trataremos de poner en evidencia. 


\section{MARCO METODOLÓGICO Y CONCEPTUAL}

\subsection{Metodología.}

En el desarrollo de este trabajo se utilizó el método analítico de la filosofía. Inscríbase esta investigación, por tanto, en el marco de una metateoría del derecho, toda vez que parte del análisis conceptual de distintos constructos teóricos que pretenden dar cuenta del fenómeno jurídico.

Por ende, haremos un análisis conceptual, primordialmente de las nociones de "neoconstitucionalismo", "derecho natural", "vetus iusnaturalismo" y de "neopositivismo". Procuraremos aclarar cada uno de los sentidos en que estos términos aparecen en la Filosofía del Derecho actual, así como los elementos que componen los enunciados básicos de las teorías que suelen ir adheridas con esos términos. Al análisis referido se le complementará la comparación entre sus elementos iusteóricos, localizando así conexiones y relaciones teóricas. ${ }^{3}$

\subsection{Marco conceptual.}

En el contexto de este trabajo, la comunidad iusfilosófica contemporánea (Carbonell, 2007; Comanducci, 2010) en general apela a tres sentidos de neoconstitucionalismo: el normativo, el teórico y el doctrinario. De esos tres sentidos, nos interesa el relacionado con el neoconstitucionalismo como teoría. En otros contextos, también se le denomina no-positivismo (Vigo, 2017; Alexy, 2013) o postpositivismo (Atienza, 2016) .

\footnotetext{
${ }^{3}$ En este sentido, podemos hacer nuestra la explicación que da el profesor costarricense Juan Diego Moya en su estudio sobre el Ente Por Sí Necesario: "Un concepto menos inclusivo y más opulento en notas intencionales es aquel según el cual analítica es toda filosofía que o bien otorga preponderancia al estudio del lenguaje [...], o bien reconoce que el examen de los sentidos y el esclarecimiento desambiguante y distintivo de las expresiones es una [condición necesaria] relativamente a toda pesquisa filosófica fértil. Desde este punto de mira, toda recta filosofía implica identificar los significados de unas ciertas expresiones, como por ejemplo las ontológicas" (Moya, 2014, p. 3). En la tradición analítica, el análisis es una actividad filosófica que puede ser entendida al menos de tres formas: (a) des-composicional o de identificación de las partes que forman un todo; (b) regresivo o de ubicación de las premisas supuestas que sustentan una conclusión y (c) traductivo o de reformulación de una tesis o expresión problemática, en una estructura en la que las dificultades ya no estén presentes (Beaney, summer, 2018).

${ }^{4}$ Atienza niega que su concepción puede denominarse neo-constitucionalista (Atienza, 2016).
}

El neoconstitucionalismo, entendido como teoría, presenta como una de sus pretensiones centrales la superación del positivismo jurídico como teoría jurídica que explique el fenómeno jurídico. Ius positivista es toda teoría del derecho que (a) asuma la separación conceptual (i.e. no necesariamente fáctica) entre derecho y moral (i.e. tesis de la separación) y (b) todo derecho es el producto contingente de fuerzas políticas, histórico-socialmente situadas (i.e. tesis de las fuentes sociales) (Guastini, 2016). La sustitución neo-constitucionalista pone como premisa primera la conexión conceptualmente necesaria entre el derecho y la moral (si bien podría no ser una identidad plena), y la posibilidad de una corrección judicial del derecho positivo. Siendo que las propias constituciones reconocen estas instancias correctivas (por medio del reconocimiento de los Derechos Fundamentales), por tanto, se requiere una teoría que explique ese fenómeno, y que, además de explicar el fenómeno, permita a su vez crear el derecho por medio del trabajo científico de los juristas y filósofos del derecho (v.g. Atienza, 2016).

Estamos ante el vetus iusnaturalismo (al que también invocaremos como iusnaturalismo tradicional), particularmente, si se afirma que la conexión entre derecho y moral se justifica en los casos en que la moral tiene como fuente la propia naturaleza de las cosas. Esta tesis suele ser de tipo aristotélico tomista, es decir, adopta una ontología realista.

Finalmente, se habla de iusnaturalismo en general en todo caso en que primordialmente se reconoce la tesis de la conexión necesaria entre derecho y moral. Esta conexión puede manifestarse o ser caracterizada de distintas maneras. Ha de tenerse en cuenta que la moral con la que el derecho -según esta tesis- tiene una conexión conceptual, no es la social e históricamente contingente, sino la crítica o correcta (Guariglia y Vidiella, 2011). En esencia, se afirma desde el iusnaturalismo, hay un derecho que trasciende (o que no se basa) en los mandatos emanados por la autoridad. 
Así entonces, procuraremos demostrar que el iusnaturalismo tradicional y el neoconstitucionalismo tienen algo en común: ambos perfectamente pueden ser considerados como pertenecientes a la gran familia de iusnaturalismos, según hemos conceptualizado este último término.

\subsection{Resultados.}

En síntesis, los resultados a los que arribaremos mediante el análisis conceptual de cada uno de los constructos ya precisados son los siguientes:

a). La determinación de que el neoconstitucionalismo es, en efecto, una especie de iusnaturalismo.

b). La distinción entre neoconstitucionalismo y otras formas de iusnaturalismo únicamente puede realizarse por vía de las diferencias específicas.

c). Lasdiferencias específicasentreeliusnaturalismo tradicional (o vetus iusnaturalismo), y el actual neoconstitucionalismo, estriban ante todo en dos cuestiones: (a) el concepto y papel que dan a la argumentación jurídica y (b) su posición sobre la ontología de los derechos naturales.

\section{DISCUSIÓN SOBRE LA TESIS CENTRAL}

Partiendo de la precisión conceptual antecedida, queda entonces establecer puntualmente que el neoconstitucionalismo y el iusnaturalismo tradicional son ambos especies del genus iusnaturalismo.

El gran tema en el que están de acuerdo, tanto Atienza como García Amado, es que lo que distinguiría a los iusmoralistas de tipo neoconstitucionalista, de los iusnaturalistas de corte tradicional, es que los últimos aceptaría un derecho natural de corte metafísico; pero metafísico entendido como trascendente del ser humano. Con ello, por tanto, sería necesaria la negación de la tesis de las fuentes sociales, tesis que asumirían los neoconstitucionalistas, y sería la tesis que permitiría (tanto a Atienza como a García Amado) afirmar que el neoconstitucionalismo no es una especie de iusnaturalismo.
La tesis del iusmoralismo podría ser precisada en el sentido que ya Maynez daba hace años en su clásico libro de introducción al estudio del derecho. En dicho libro introductorio, Maynez afirmaba que se suele llamar derecho natural a aquel que remite a un orden intrínsecamente justo "que existe al lado o por encima del positivo"5 (Maynez, 2002, p. 40).

Es de nuestra consideración que esta definición no es muy distinta de la que presenta García Amado respecto de los iusmoralismos. Según García Amado, se habla de iusmoralismo cuando se "afirma que las normas de la moral verdadera u objetivamente correcta forman parte del Derecho mismo" (García Amado, 2014,.).

Ambas definiciones coinciden en un punto muy importante: que hay algo "más allá" del derecho postulado por una autoridad histórica. Quizás, 1o que habría que tomar con cuidado es esta noción de más allá, que el mismo Maynez reconoció que puede entenderse de diversas maneras, y que no en todas ellas hace referencia a un mundo trascendente ${ }^{6}$ (García Maynez, 2002, p. 41-44) ${ }^{7}$.

En este contexto, decir que hay un derecho más allá del positivo, implicaría aceptar (como lo afirma García Amado con su noción de iusmoralismo) que la moral (crítica) sería parte de ese "más allá" que podría no postular el autor del derecho positivo. Así entonces, debemos poner énfasis en la tesis según la cual la esencia de la concepción ius-naturalista se encuentra en las ideas de que hay un derecho más allá del positivo y / o que existe un derecho a la par

\footnotetext{
${ }^{5}$ También, hay toda una historia sobre el iusnaturalismo en Goldschmidt (Goldschmidt, 1987). Hemos optado citar a Maynez por la influencia que ha tenido también en la pedagogía de diversas generaciones juristas en Latinoamérica. Sorprende que, a pesar de conocer estos antecedentes, se insista que el concepto de iusnaturalismo remite a una sola escuela y a una sola noción de derecho natural.

${ }^{6}$ Maynez reconoce que existen diversos sentidos de la palabra natural y que a partir de esas definiciones se pueden distinguir diversas clases de teorías ius-naturalistas. Algunos de esos sentidos son los siguientes: sentido biológico -que, dicho sea de paso, es puramente físico y nada tiene que ver con el trascendente que le adjudican tanto Atienza como García Amado-, teológico, naturaleza social o concepción sociológica del derecho natural, racionalista, objetivista, etc. (García Maynez, 2002, p. 41-44).

${ }^{7}$ En Maynez, cuando se habla de derecho positivo, quiere decirse del vigente o incluso del eficaz (incluyendo así la costumbre).
} 
del positivo. Tal sería el género de toda una familia de teorías que admiten la existencia de un orden jurídico no puesto por autoridad alguna y que de alguna manera sirve, ora para fundamentar el derecho, ora para corregirlo, ora para ambas empresas.

El neoconstitucionalismo, tal como se definió, admite la posibilidad de corrección del derecho emanado de la autoridad histórica (que en nuestros ordenamientos es una autoridad legitimada democráticamente). Esa corrección, como bien señala García Amado, viene dada, no en el derecho mismo, sino en una moral que debe ser entendida objetiva y universalmente válida. Siendo esto así, no vemos diferencia alguna con el vetus iusnaturalismo, el cual parte de la misma tesis. De otra forma: no se sustenta la tesis según la cual el neoconstitucionalismo no forma parte del género iusnaturalismo. Es cierto que podrían presentarse algunas diferencias entre el iusnaturalismo tradicional y el neoconstitucionalismo. Empero, dichas diferencias que no permitirían afirmar que el neoconstitucionalismo no sea una especie de la gran familia de iusnaturalismos que han aparecido a lo largo de la historia de la Filosofía del Derecho.

Dado que se afirma la existencia de contenidos jurídicos que trascienden lo mandado por la autoridad, tanto en el neoconstitucionalismo como en el iusnaturalismo tradicional se requieren algunas condiciones para la determinación de los contenidos de esos mandatos que trascienden lo ordenado por la autoridad. Para realizar dicha determinación, se encuentra necesario un método para establecer (o conocer) sus contenidos; requieren partir de un objetivismo moral (pues de lo contrario, no se sostiene la doctrina de los Derechos Fundamentales).

\section{LA PRÁCTICA JURÍDICA SEGÚN EL IUS- NATURALISMO}

\subsection{Silogismo y argumentación.}

Tanto en el iusnaturalismo tradicional como en el postpositivismo se acepta que es posible conocer algún sentido de justicia. Sin embargo, el modo de llegar a él es distinto. En el caso del postpositivismo, se entiende que la argumentación jurídica funge como método para conocer los contenidos de la moral objetiva. En este asunto, estas concepciones contemporáneas difieren difiere del iusnaturalismo tradicional ${ }^{8}$.).

En cambio en el vetus iusnaturalismo, conocer las esencias de las cosas (a partir del realismo de las esencias) permite el conocimiento sobre su naturaleza. Mediante ese conocimiento, se pueden construir, por medio de silogismos, una serie de verdades que, toda vez que se parta de premisas verdaderas, habilitan a inferir proposiciones verdaderas. Esta forma de operar da una mayor importancia a la deducción y permite afirmar que se tiene auténtico conocimiento.

En el iusnaturalismo tradicional no es que se niegue la retórica, simplemente es subsidiaria: únicamente aplica cuando, por alguna razón, no se tenga todavía conocimiento pleno de una cierta realidad jurídica. He ahí, su cercanía con la retórica. Pero de igual manera, en el neoconstitucionalismo la argumentación se complejiza a su vez con otras técnicas, como las de la ponderación.

La principal distinción entre ambas escuelas en cuanto al método de abordar el conocimiento de los valores es en cuanto a la distancia que toman con relación a la importancia que se le presta a la deducción para concluir las verdades morales.

\subsection{Objetivismo moral mínimo y el problema de las fuentes sociales.}

Dado que el iusnaturalismo tradicional afirma la existencia de esencias, el conocer los aspectos accidentales de un ente, como su naturaleza jurídica, no es un problema irresoluble. En cambio, lo interesante del neoconstitucionalismo es afirmar que no existen las esencias (tesis nominalista) (Nino, 2006) y sin embargo afirmar que hay una moral crítica objetiva (ya sea mínima) proveniente de las fuentes sociales del derecho.

\footnotetext{
${ }^{8}$ En el caso de los predecesores de la argumentación, como en Perelman o en Toulmin, según lo entiende Atienza (Atienza, 2013), la deducción no tiene un papel distinguible, dándole énfasis a la retórica. Mientras que en Alexy y Atienza la deducción tiene su importancia dentro del enfoque formal de la argumentación (Atienza, 2013)
} 
Para Atienza, esta es una cuestión primordial, dado que su objetivismo moral estriba en que este es perfeccionable. En cambio, el "objetivismo moral máximo" (que llama absolutismo moral), no lo sería por tanto se equipara con el del realismo. Consideramos que Atienza en este punto no brinda razones para tal afirmación (Atienza, s.f.) ${ }^{9}$.

El iusnaturalismo tradicional aristotélico tomista en verdad lo que dice es que el derecho natural, más que estar en un mundo trascendente (cosa que no admite), está en la naturaleza de las cosas mismas. He ahí la inspiración aristotélica, en particular la doctrina hilemórfica de los objetos. Dicho en términos aristotélicos, uno de los accidentes de las cosas es la posesión de una naturaleza jurídica. Una ley positiva que no se ajuste a esa exigencia jurídica que tiene la cosa en sí, es una ley "desnaturalizada". Pero nunca afirma que el derecho natural está fuera de este mundo, en un ámbito eterno. Esta afirmación únicamente cabe para los platónicos quienes asumían la tesis de la realidad de las ideas fuera de este mundo. En cambio, en el caso de la teoría iusnaturalista aristotélico tomista, un derecho que no sea el natural únicamente remite a las nociones de derecho divino (que no es natural y, por tanto, únicamente conocido por medio de las escrituras y la fe; y de alguna manera no vinculante para los paganos ${ }^{10}$ ) y de derecho eterno.

En este contexto, las fuentes sociales solo son entendidas como parte de lo real. Las fuentes sociales son parte del mundo social que es conocible por vía exclusiva de la razón humana y que por tanto prescinde de toda revelación. En todo caso, lo que existe es una pugna entre lo que la autoridad afirma (como fuente social) y lo que la naturaleza de las cosas parecería indicar. Pero, esta misma situación se presenta con el neoconstitucionalismo actual, solo que planteada en otros términos: habría en efecto un derecho que impone la autoridad democrática, pero éste es

\footnotetext{
${ }^{9}$ Baste pensar en un debate como el de la esclavitud: si bien desde el derecho natural se planteó como parte de la naturaleza de las cosas, no menos cierto es que el mismo derecho natural reconoció luego que había sido una manera equivocada de concluir. De forma tal, que de ninguna manera puede afirmarse que los contenidos del derecho natural siempre fueron considerados eternos

${ }^{10}$ En este sentido, piénsese en la razón para que Tomás de Aquino planteara la Summa contra gentiles.
}

86-|Revista de Derecho. Vol. 40. No. 1. Año 2018 cuestionable a partir de la argumentación jurídica, por medio de la cual conoceríamos las respuestas correctas (en lugar de hacer referencia a las cosas).

La afirmación de García Amado citada en la introducción de este trabajo, en la que se plantea dónde se puede obtener el conocimiento acerca del derecho natural, es completamente legítima: según el iusnaturalismo tradicional, la remisión sería a la naturaleza misma de las cosas. En cambio, los neoconstitucionalistas tendrían que admitir que sus conclusiones no son más que el resultado de un ejercicio dialógico intersubjetivo, pero difícilmente objetivo, toda vez que es sólo intersubjetivo. En el caso del objetivismo moral mínimo de Atienza (y que es asumido básicamente por otros post-positivistas importantes (v.g. Alexy, 2013)), más que prestar atención a las cosas, se supone que ese conocimiento de los contenidos de la moral objetiva se obtiene de la pura dialéctica entre los participantes de un diálogo racional. En este sentido, la moral objetiva más bien parece subjetiva, puesto que no depende de las cosas (objetividad), sino de lo que los sujetos puedan acordar (o consensuar) por vía del debate ideal.

\section{SU CONTRASTE CON EL NEOPOSITIVISMO}

Para ver con más claridad lo antes expuesto, consideramos compararlo con lo que podríamos llamar un neopositivismo jurídico, la cual no es más que una posición hipotética ${ }^{11}$. El neopositivismo -proponemos- es la tesis según la cual el derecho lo constituyen, no sólo las normas (en cuanto reglas y principios) que emanan de la autoridad jurídica ordinaria, sino además las decisiones de los tribunales en materia de Derechos Humanos (en adelante DDHH). Es decir, si un tribunal no ha delimitado el sentido de un texto de DDHH no se puede afirmar que existe un cierto derecho, o, más exactamente, no puede conocerse la respuesta correcta para resolver un caso que requiera la delimitación de los sentidos de un texto de DDHH (v.g. Guibourg, 2013, cap. 4).

\footnotetext{
${ }^{11}$ En la historia del pensamiento occidental, neo-positivismo es el nombre de un corriente de epistemología y filosofía de la ciencia que existió -aproximadamente- entre 1920 y 1950. Tesis características del neo-positivismo fueron que el conocimiento seguro se obtiene por medio de la observación de hechos positivos, la unidad formal de todas las ciencias, el verificacionismo como criterio de significado de los enunciados, entre otras ideas. En el texto principal utilizo la expresión, pero no aludo a este movimiento.
} 
Así entonces, lo que vendría a distinguir al neopositivismo del neoconstitucionalismo estribaría en el abordaje que se da al problema de la respuesta correcta. Para el primero, la respuesta correcta sólo puede venir directamente de la decisión del órgano decisor de última jerarquía, lo que conlleva que no habría más derecho que el determinado por este órgano. Incluso, tal posición podría suponer que no hay respuestas correctas más allá de lo que señalara el último decisor.

Los neopositivistas admitirían lo primero: no habría más derecho que el determinado por el órgano decisor. Los neoconstitucionalistas tendrían que adherirse a lo segundo: que hay respuestas correctas más allá de lo que señalara el último decisor. Estos neoconstitucionalistas, tal como hemos venido demostrando, de vuelta tienen que afirmar que hay un "más allá", siendo irrelevante si este más allá es una moral objetiva (como denuncia García Amado con su noción de iusmoralismo) o que esta fuente venga de la propia naturaleza de las cosas (iusnaturalismo tradicional).

De forma tal que, para los neopositivistas, se desplaza la autoridad por antonomasia creadora del derecho -el Poder Legislativo- a la Corte como último decisor ${ }^{12}$. En cambio, los neoconstitucionalista siguen asumiendo que existe "una más allá del Derecho correcto", que puede ser también entendido "un más allá de lo que las cortes afirmen”.

El paleopositivismo (según le llama Ferrajoli al ius-positivismo de Kelsen y Hart) y el neopositivismo comparten la tesis según la cual todo derecho válido es el declarado por una autoridad (que ostenta la competencia y los procedimientos para efectuar dichos actos). Lo que cambia en cada respecto, es que mientras el paleopositivismo considera a la autoridad legislativa como la autoridad creadora de derecho por antonomasia, el neopositivismo considera como la autoridad de los contenidos jurídicos a los jueces, en particular a los jueces de Cortes Constitucionales, e, incluso, de los Órganos Internacionales de Derechos Humanos.

${ }^{12}$ Como ha llamado la atención Guibourg (Guibourg, 2005), de alguna forma esta tesis ya se encontraba en la Teoría Pura del Derecho de Hans Kelsen.
Esta lectura de las cosas nos permite hacer la siguiente afirmación: tanto los iusnaturalistas tradicionales como los neoconstitucionalistas admitirían que existe la posibilidad de que los últimos decisores se equivoquen en el derecho por razones morales objetivas y, por tanto, que haya contenidos jurídicamente válidos que los decisores no tomen en cuenta, nuevamente, por razones morales objetivas. Si esa decisión es extremadamente injusta, pueden concluir que no es derecho.

\section{CONCLUSIONES}

- Por el panorama así descrito, queda más claro que entre el neoconstitucionalismo y el iusnaturalismo tradicional hay más puntos de contacto que de divergencia. Esto permite sustentar el clasificar al neoconstitucionalismo como una especie de iusnaturalismo. Nuestra principal consideración para criticar tanto la posición de Atienza como la de García Amado con respecto a la supuesta distinción entre iusnaturalismo $\mathrm{y}$ neoconstitucionalismo es el concepto de iusnaturalismo que asumen, pues es a todas luces paupérrimo, toda vez que siempre se reconoció la gran diversidad de escuelas de derecho natural a lo largo de la historia.

- El otro gran problema es haber considerado que tanto el iusnaturalismo en general, como el iusnaturalismo aristotélico tomista, niegan la tesis de las fuentes sociales. En realidad, lo que postulan es la posibilidad del conflicto entre la naturaleza normativa de las cosas y las pretensiones de la autoridad; al ser demasiada la desarmonía entre ambos aspectos, se podría decir que lo creado por las fuentes sociales no es derecho. Pensamos, tal como ya señalamos, que esa tesis también es compartida, aunque con otro lenguaje, por el neoconstitucionalismo.

- Por su parte, se ha distinguido entre neoconstitucionalismo y neopositivismo jurídico. Mientras el neoconstitucionalismo reconoce que habría un derecho más allá incluso de lo que 
los tribunales aceptarían, el neopositivismo no: derecho sería únicamente aquello que afirman los tribunales de última instancia.

- Por otra parte, vale reconocer las distinciones que se presentan entre el neoconstitucionalismo y el vetus iusnaturalismo. Mientras en el iusnaturalismo, tanto aristotélico-tomista como en el racionalista moderno e ilustrado, el método deductivo es el utilizado por excelencia, en el actual neo-constitucionalismo (que Atienza prefiere llamar post-positivismo), la argumentación funciona para tales fines ${ }^{13}$.

- Así entonces, podemos afirmar que no todo iusnaturalismo fue aristotélico-tomista y que por tanto la escuela aristotélica-tomista no fue la única escuela de derecho natural que existió en la historia de la cultura jurídica occidental. Hubo muchas maneras de entender el término "natural", lo que de ahí devino la gran cantidad de escuelas de iusnaturalismo. Y, tal como hemos sostenido, a partir de todas estas aclaraciones $\mathrm{y}$ dadas sus características, el neoconstitucionalismo perfectamente es entendible como una nueva escuela de derecho natural.

\section{BIBLIOGRAFÍA}

- Alexy, R. (2013). El no positivismo incluyente. DOXA, Cuadernos de Filosofía del Derecho, núm. 36, 15-23.

- Atienza, M. (2013). Curso de argumentación Jurídica. Madrid. Trotta.

\footnotetext{
${ }^{13}$ El método deductivo de tipo silogístico -sobre todo si se conecta con un realismo ingenuo en ontología- pretendidamente habilita a que, por medio de los conceptos plasmados en juicios y estos a su vez organizados en un razonamiento, se obtendrían conclusiones importantes: un nuevo conocimiento, cierto y verdadero. En la argumentación jurídica, por su parte, la discusión sobre el papel de la deducción debe compartir protagonismo con los enfoques materiales y pragmáticos de la argumentación en el que se agregan otros elementos como la fuerza emotiva de ciertos términos que pueda conmover a la audiencia, tener presente la audiencia misma para convencer, etc.
}

- Atienza, M. (2016). Ni positivismo jurídico ni neoconstitucionalismo: una defensa del constitucionalismo postpositivista. En: Lorenzo Peña, Txetxu Ausín. Díez Conceptos y Valores Constitucionales (29-58). Madrid. Plaza y Valdéz.

- Atienza, M. (s.f.). Objetivismo moral y derecho. Obtenido de https://dfddip.ua.es/es/ documentos/objetivismo-moral-y-derecho. pdf? noCache $=1458554296851$

- Atienza, M., \& García Amado, J. A. (2016). Diálogo entre Manuel Atienza y Juan Antonio García Amado. Diálogos Jurídicos: Anuario de la Facultad de Derecho de la Universidad de Oviedo, num. 1, 229-255.

- Beaney, M. Philosophy (Summer 2018 Edition). Analysis. En: Edward N. Zalta, The Stanford Encyclopedia of. Obtenido de URL $=\underline{\text { https: } / /}$ plato.stanford.edu/archives/sum2018/entries/ analysis/

- Carbonell, M. (2007). El neoconstitucionalismo en su laberinto. En: M. Carbonell, Teoría del Constitucionalismo (págs. 9-12). Madrid. Trotta.

- Comanducci, P. (2010). Hacia una teoría analítica del Derecho. Madrid. Centro de Estudios Políticos y Constitucionales.

- Comanducci, P.(2016). Estudios sobre Constitución y Derecho Fundamentales. Querétaro. Instituto de Estudios Constitucionales de la Ciudad de Querétaro.

- García Amado, J. A. (9 de octubre de 2014). Iusmoralismos y respuestas correctas. Recuperado el 2019, de Dura Lex. Política, Derecho, Sociedad. Y algo de otras cosas: http://garciamado.blogspot. com/2014/10/iusmoralismo-y-respuestascorrectas.html

- García Maynez, E. (2002). Introducción al estudio del derecho. México D.F. Porrua. 
- Goldschmidt, W. (1987). Introducción filosófica al derecho (6ta edición 4 a reimpresión ed.). Buenos Aires. Depalma.

- Guariglia, O. \& Vidiella, G. (2011). Breviario de ética. Buenos Aires. Edhasa.

- Guastini, R. (2016). Sintaxis del derecho. Madrid. Marcial Pons.

- Guibourg, R. (2005). Derecho, Sistema y Realidad. Buenos Aires. Astrea.

- Guibourg, R. (2013). Saber Derecho (1a ed.). Buenos Aires. AbeledoPerrot.

- Moya Bedoya, J. D. (2014). De la existencia del ente por sí necesario. Conceptos y argumentos expuestos de acuerdo con la concepción analítica de 'filosofía'. Texto no publicado facilitado por el autor.

- Nino, C. S. (2006). La validez del derecho. Buenos Aires. Astrea.

- Vigo, R. L. (2017). La interpretación (argumentación) jurídica en el Estado de Derecho Constitucional. Ciudad de México. Tirant lo blanch. 